\title{
An Approach to Establishing Simultaneous Server-Side Connections for NFC/Bluetooth Enabled Quiz Management Systems
}

By Jedidiah Aqui \& Michael Hosein

University of the West Indies

Abstract- Near Field Communication is a set of communication protocols for communication between two electronic devices over a distance of $4 \mathrm{~cm}$ or less and Bluetooth is a wireless technology standard used for exchanging data between fixed and mobile devices over short distances using UHF radio waves in the industrial, scientific and medical radio bands, from 2.402 $\mathrm{GHz}$ to $2.480 \mathrm{GHz}$, and building personal area networks(PANs). Both these protocols facilitate wireless/ internet less communication between devices that have the capabilities. This paper seeks to further expand the concept and usage of these protocols via the examination of modifications made to a Quiz Management System. The Quiz Management System, which is a solution to further advance the notion of a smart classroom setup within the context of shortrange wireless technologies has already seen iterations developed on both the NFC and Bluetooth side of things.

Keywords: quiz management system, smart classroom, protocol, bluetooth, NFC, UHF. GJCST-A Classification: C.2.1

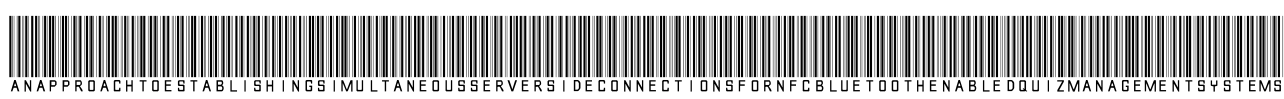

Strictly as per the compliance and regulations of:

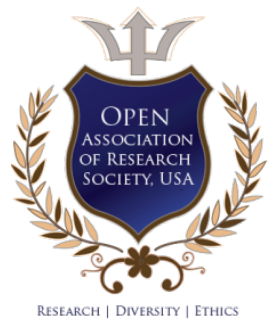

(C) 2020. Jedidiah Aqui \& Michael Hosein. This is a research/review paper, distributed under the terms of the Creative Commons Attribution-Noncommercial 3.0 Unported License http://creativecommons.org/licenses/by-nc/3.0/), permitting all non commercial use, distribution, and reproduction in any medium, provided the original work is properly cited. 


\title{
An Approach to Establishing Simultaneous Server-Side Connections for NFC/Bluetooth Enabled Quiz Management Systems
}

\author{
Jedidiah Aqui ${ }^{\alpha} \&$ Michael Hosein ${ }^{\sigma}$
}

\begin{abstract}
Near Field Communication is a set of communication protocols for communication between two electronic devices over a distance of $4 \mathrm{~cm}$ or less and Bluetooth is a wireless technology standard used for exchanging data between fixed and mobile devices over short distances using UHF radio waves in the industrial, scientific and medical radio bands, from $2.402 \mathrm{GHz}$ to $2.480 \mathrm{GHz}$, and building personal area networks(PANs). Both these protocols facilitate wireless/ internet less communication between devices that have the capabilities. This paper seeks to further expand the concept and usage of these protocols via the examination of modifications made to a Quiz Management System. The Quiz Management System, which is a solution to further advance the notion of a smart classroom setup within the context of short-range wireless technologies has already seen iterations developed on both the NFC and Bluetooth side of things. The QMS will be further developed to address a key limitation that was observed. That is the inability to facilitate multiple serverside connections to client devices beyond the established limit of the existing model (limit being 5 simultaneous connections at a time). This is important due to the fact that, in addressing this limitation, the adoptability and feasibility of this system would grow substantially as persons utilizing the system would not be impeded by the inefficiencies of having to walk and connect to a single device at a time or five (5) devices (maximum) at a time within a university classroom setting, and by 'university classroom' setting, one could imagine a setup such as a class of over two hundred(200+) students.
\end{abstract}

Keywords: quiz management system, smart classroom, protocol, bluetooth, NFC, UHF.

\section{INTRODUCTION}

W ith the advent of a global pandemic, societies at large have witnessed the shutting down of numerous institutions of gathering and socializing. Many of those institutions consist of churches, sporting events, entertainment events, schools, etcetera. In light of these shutdowns, governments the world over, were forced to adapt and facilitate numerous activities that were once viewed as "in person" or "attendance mandatory" activities via the internet and its many online portals. The area in which this project seeks to primarily address, is the educational institutions (Schools).

Author a $\sigma$ : Department of Computing and Information Technology, University of the West Indies, St Augustine, Trinidad.

e-mail:michael.hosein@sta.uwi.edu
The use of online classes is not a new topic of discussion or concept as far as technological advances in the educational sphere is concerned. Numerous papers and contributions have been made with respect to online classes/sessions (Kulkarni et al., 2013 and Frydenberg, 2007) and (Grandon, Alshare, and Kwun,2005).Additionally, what is quite notable in the midst of an ongoing pandemic is the vast migration of many institutions to the use of Online platforms to host their classes, meetings, seminars etcetera. The fastpaced transformation of these institutions to technological platforms all encompass aspects of what is being termed the "new normal". But one must also take into consideration the latter phases of the "new normal", which would encompass a steady and careful re-engagement of the former way of life at least to a substantial extent. With a re-engagement of the former, one can expect institutions of learning to begin the process of re-opening and facilitating in-person knowledge transfer activities such as classes, lectures, tutorials, etcetera as safely as possible. With safety being of paramount priority, one can expect all necessary guidelines as far as social distancing dictates, to be enforced.

To address the adaptation to the latter phase of the "new normal" the option of a smart classroom approach would be explored and further elaborated upon. (According to Shalini, December 2018) A smart classroom is where the concept of blended learning is considered to be blooming, it isa technologically enhanced environment that enables teaching and learning opportunities on a different level. Within the context of a smart classroom, the use of the Quiz Management System(QMS) would come into play. To generalize, the QMS is a system that is geared towards facilitating the distribution of quizzes and/or quiz material by the teacher(server) as well as subsequent retrieval of quiz material by students (clients) for grading and redistribution.

The QMS itself is a step in the right direction as it brings the notion of leveraging Bluetooth/NFC technology to further promote or simulate a smartclassroom setup. The QMS captured functionality that allowed students to submit their work and review the marked material and it also allowed the teachers(server)to select the quiz material to distribute, 
collect the quiz material for marking and perform analytics on the data obtained before subsequently distributing the marked material. Its merits cannot be overlooked as it fostered contactless communication from client to server devices and vice versa. However, there were some limitations in the developed model that were noted.

A key limitation of the QMS was its inability to support a large number of simultaneous connections on the server side. Consider the work done by (M.Hosein and L.Bigram, 2013). Theirs was a Bluetooth Quizzing Application in Android called Blue Q. The developed application was able to simulate much of the QMS's core functionality via the use of the Bluetooth protocol, however it was only able to facilitate simultaneous connections to a maximum of 5 devices, the authors further postulate that future work on the developed application could entail the supporting of more simultaneously Bluetooth connections on the server side. Additionally, in the studies done by (Mohammed Salah Abood, Mahamod Ismail, and RosdiadeeNordin, 2017). The authors were able to develop and further add to the work of the Bluetooth application formerly mentioned by utilizing XML to structure messages passed between both client and server. Furthermore, in their work, the authors developed a solution using Near Field Communication Technology (NFC) as the main protocol for transferring information from Client(student) to Server(Teacher). However as was the case in the first iteration of the application, this was also a system developed on the basis of one to one communication between Client and Server devices.

This problem can further be grasped by adding some context. For example, if the QMS was to deployed in a classroom of 5-6 persons, sharing course content to students and also receiving course content/solutions from students(client), would be considered a relatively simple task that can be performed in a short timeframe, however in the context of a University classroom that may house well over 100-200+ students in a session. Distributing course contents(quizzes) and receiving student feedback can pose quite a challenge, as one must now consider the amount of time it takes to fully conduct this activity from the Server to each individual Client, given that there are no anomalies to hinder the process with each student.

An additional level of analysis is also required in establishing which of the two protocols (NFC or Bluetooth) should the testing of multiple server-side connections be executed with. In retrospect, whilst these developed models did in fact have a substantial impact in promoting a smart classroom setup using Bluetooth and NFC technology, the identified gaps would serve as an indication as to the slow adoption of the "smartclassroom" concept which sees the utilization of these protocols for distributing information. It is not so much so as to how to develop NFC/Bluetooth solutions to compliment the idea of a smart classroom setup, but is a matter of how do we increase the efficiency and practicality of these systems to gain further support from Universities the world over, in the midst of a pandemic. This project entails the resolution of these core problems as an iterative step to the already developed Quiz management system. By addressing these problems, the feasibility of a safe smart classroom environment in the "new normal" period can be further bolstered and gain support.

\section{il. Literature Review}

In This Chapter, we would not only be exploring the research work and implementations conducted as it relates to similar quizzing applications. But we will also be exploring the work done as it pertains to the concepts of Bluetooth connections to multiple devices as well as identify the merits and limitations of each of the discussed material.

(Mohammed, Mahamod, and Nordin, 2016) presented an NFC-based classroom tool known as the 'Quiz management system'. The solution (QMS) was able to further advance the notion of a smart classroom setup within the context of short-range wireless technologies. As it pertains to this system, one would recognize the function of two key actors, one being the Student (Client) and the other being the Server(Teacher). For the sake of simplicity we would refer to the Student/Client device as Participant1 and the Server/teacher device as Participant 2. The core functions of each actor are as follows:

Participant 1: Is the Client/Student who would do the requesting of quizzes as well as the submission of quizzes for correction.

The key activities are captured in the below bullet point listing

1. Interacting with the $\mathrm{UI}$ of the application

2. Quiz handout activity

3. Answer Collection Activity

4. Quiz Submission Activity

5. Results Activity

Participant 2: Is the Server/Teacher who would be responsible for distributing the quizzes via his/her interface. The Server/Teacher would also be privy to additional functionality as opposed to the client and thus, can be viewed as a super-user within the context of the system.

The key activities are captured in the below bullet point listing

1. Interacting with the UI of the application

2. Input activity

3. Quiz reception activity

4. Quiz performance activity

5. Answer submission activity 
The roles of 'Participant 1' and 'Participant 1' System via the below general architecture diagram in can now be observed within the context of the QMS (Figure 2.1):

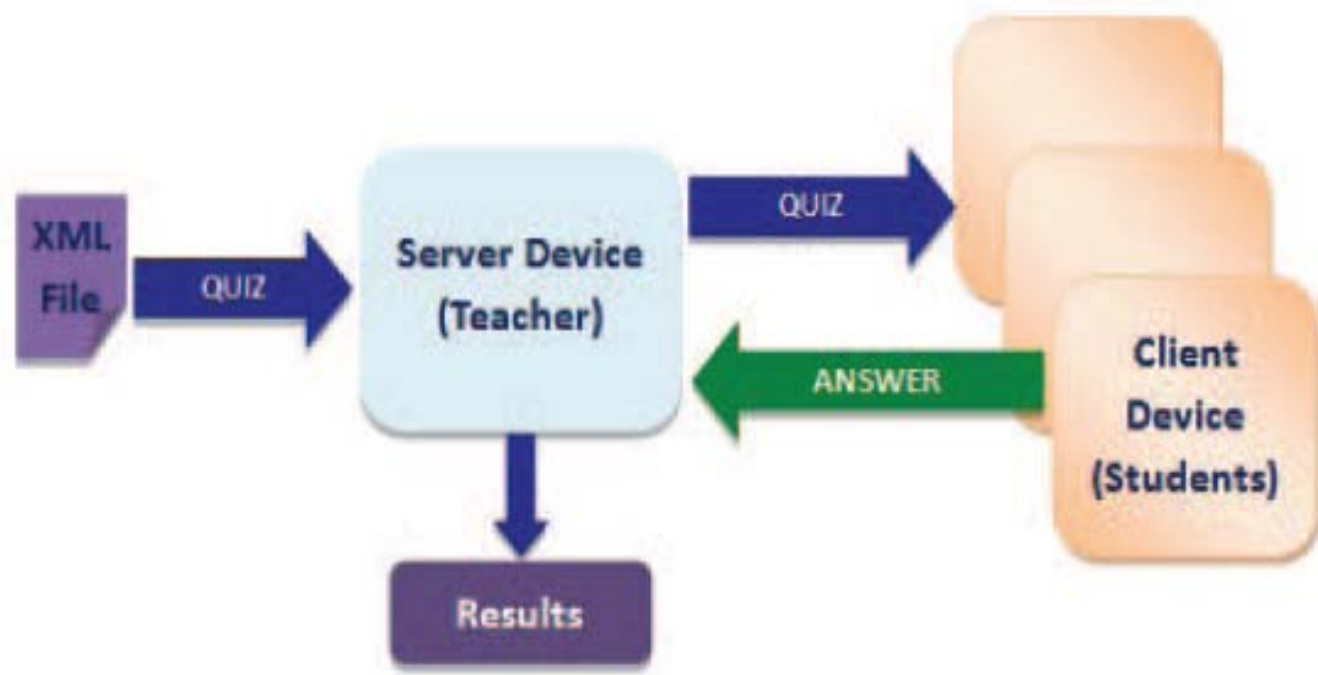

Another system was also developed by (Michael and Laura, 2013) which was a Bluetooth quizzing application for the android platform was developed. This system was quite similar to the formerly mentioned NFC solution with one of the major differences being the choice of protocol for communication and sharing of data. The protocol utilized was the Bluetooth protocol. The designed solution addresses the below problems directly:

1. Low attendance rate

2. Low pass rates

3. Poor Learning curve of students

4. Time consuming nature of a paper-based quizzing system

The solution Blue $Q$ consisted of 2 main components, which were the 'Server Side' and the 'Client Side' these components were implemented on the Android Operating System. The proposed system encompassed several key functions/features to facilitate the distribution and receiving of course content (quiz material). The core functions are as follows:

1. Start Server - This function involved starting the server

2. Manager - This function involved the creation and editing of new courses, quizzes and students

3. Data Analysis - This function involved analysis of submitted quiz material

4. Exit 


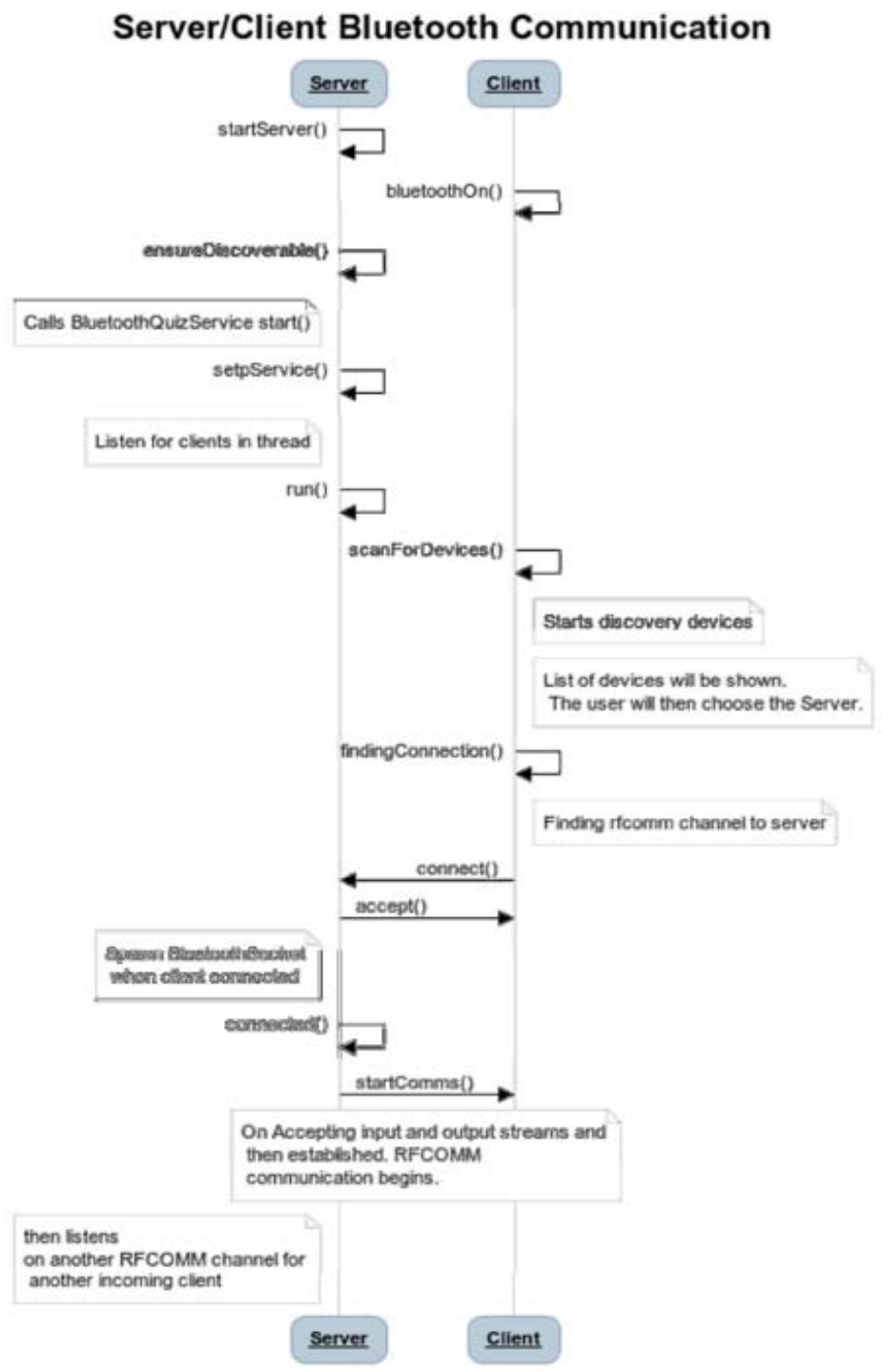

Figure 3: Showing the Core functions sequence diagram of the Blue $Q$ quizzing solution

In the work of (Brian, 2014) a low-level vantage point of establishing Bluetooth connections amongst android devices is explored and segments of code provided to further solidify the concepts. This paper was able to shed light on many of the concepts that were required for developing our solution as well as the limitations of the selected protocol for communicating data(Bluetooth). The author postulates that the Android device will be communicating with a Bluetooth module connected to an Arduino Uno microcontroller as shown in Figure 4 below. The author focused primarily on the establishment of Bluetooth connections between two devices and delved further into the issues commonly experienced throughout the process of turning on Bluetooth, establishing a connection between devices, multi-threading and transferring data.

\section{Preliminary Hardware / Software}

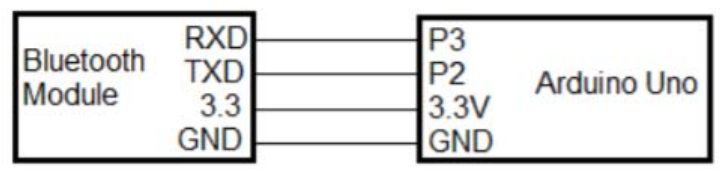

Figure 4: Showing Bluetooth Module - Arduino Uno Connectivity

Another paper done by (Naser Hossein, Motlagh (May 2012)) focused on a technical overview of Near Field communication technologies. The overview covered multiple aspects of the emergent NFC technology such as: 'Introduction to wireless communication', 'RFID technology overview', 'Physical Principles and Electromagnetism', 'Radio Frequency and Data transmission', 'NFC Applications' and 'NFC 
Security'. Pertaining to work done with respect to NFC applications, aspects of NFC that were currently being applied in the field of short-range applications were highlighted. The focus was made to the usage of NFC chips with mobile phones. In the work done, the author made mention of the three main operating models of $\mathrm{NFC}$, with the primary focus in the interest of this paper, being related to the "peer to peer communication" operating model(O.M.). It was noted that in this O.M. only two devices at a time could have exchanged the data at the link layer level.

And finally, in the work done by (Zimbric, Oct 2012) the focus was on pairing multiple devices to a Bluetooth accessory. The concept was very similar to the work done on this thesis however, the approach was very different as pairing was done based on device profiles for example 'hand-held' device or 'wireless headphones'. Furthermore, this approach primarily focused on the Bluetooth accessory as opposed to the device in which it would have been interacting with. The goal was to have the accessory connected to two devices at a time where by functions could have been executed.

\section{iil. Application Details}

There were 3 main approaches employed in the proposed solution to solve the initially identified problems. They are detailed below:

Approach 1: Version 2 (multi-channel - Identical UUIDs) This approach explored the route of adding additional RFCOMM channels that are associated with the same UUID. It mimicked the concepts of Client/Server TCP/UDP communications (Lei Wang et al.,2000)having the server socket wait for connection from a client socket, whilst a listening socket is activated for receiving new connections and mapping unto the server socket. In this concept, if we were to assign maximum of 2 channels per UUID, we would accomplish simultaneous connections for data transfer to $2 \times 8$ client devices at a time. The below Sequence Diagram Shows the flow of events in Approach 1- Version 2:

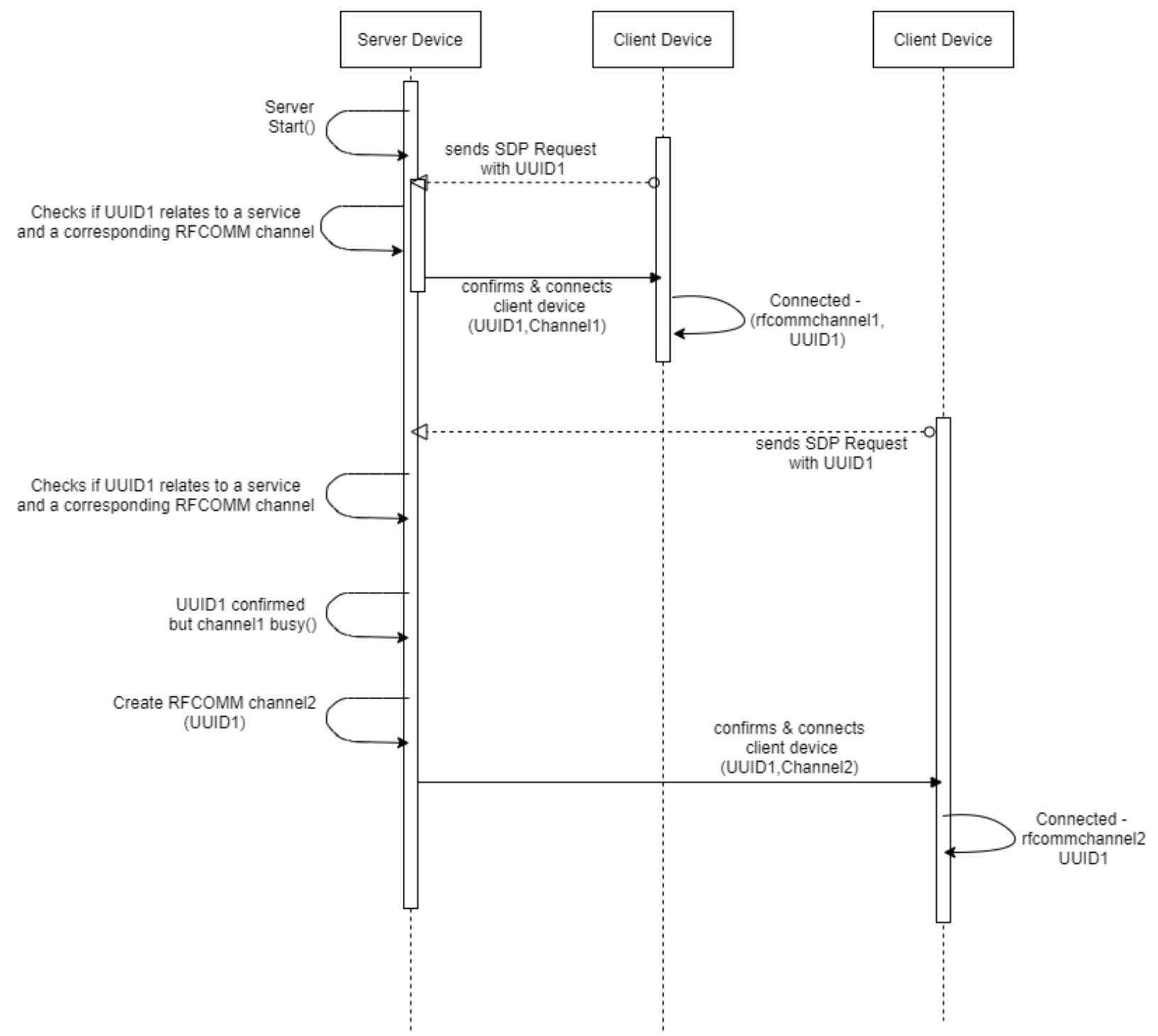

Figure 5: Sequence Diagram - Approach1 Version2 
By merit of how RFCOMM works it is stated that it would support a total of 60 simultaneous connections based on the UUIDs assigned to each of those channels. This UUID is what the client uses to firstly identify the channel it wishes to connect to via its SDP call, then a connection is established, but since RFCOMM is a serial communication protocol, it would only allow 1 connection at a time per channel, there is no multiplexing unless you switch from a serial protocol to a parallel protocol. Going further into this, the proposed modification could see the sharing of UUIDs for 2 channels at a time, 2 channels being the upper limit. Given that all the necessary provisions are made available, and the user has the optimum storage and processing power to host 60simultaneous connections, this method of dual channeling per UUID could possibly push the amount of simultaneous connections to "120"total. Now this is of course the perceived amount of simultaneous connections, however it would still be recorded as 60, since half the figure represents Unique (UUIDs) and the other a replication.

But that is observing from a holistic point of view in accordance with the protocol's specifications. As we apply this logic to the presented solution, we can now seek to address the inefficiency of having limited amount of channels used at any given time for material to be distributed by the server device or submitted by the client device. Consider the current application's limit of 8 channels per server device. That alludes to the point that there are 8 UUIDs that are hardcoded into the lowlevel code of the server device by which the RFCOMM channels can be accessed by. The solution being proposed now assigns a total of 2 channels per UUID. This therefore raises the amount of allowable connections to the server by +8 giving a total of 16 connections at a time per server device. The below figure shows the results of sharing UUIDs as more than the maximum limit of 8 devices were able to connect.

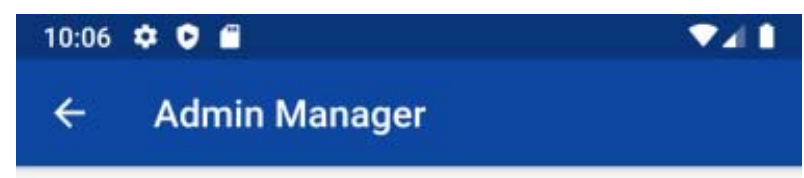

Connected Devices - (9)

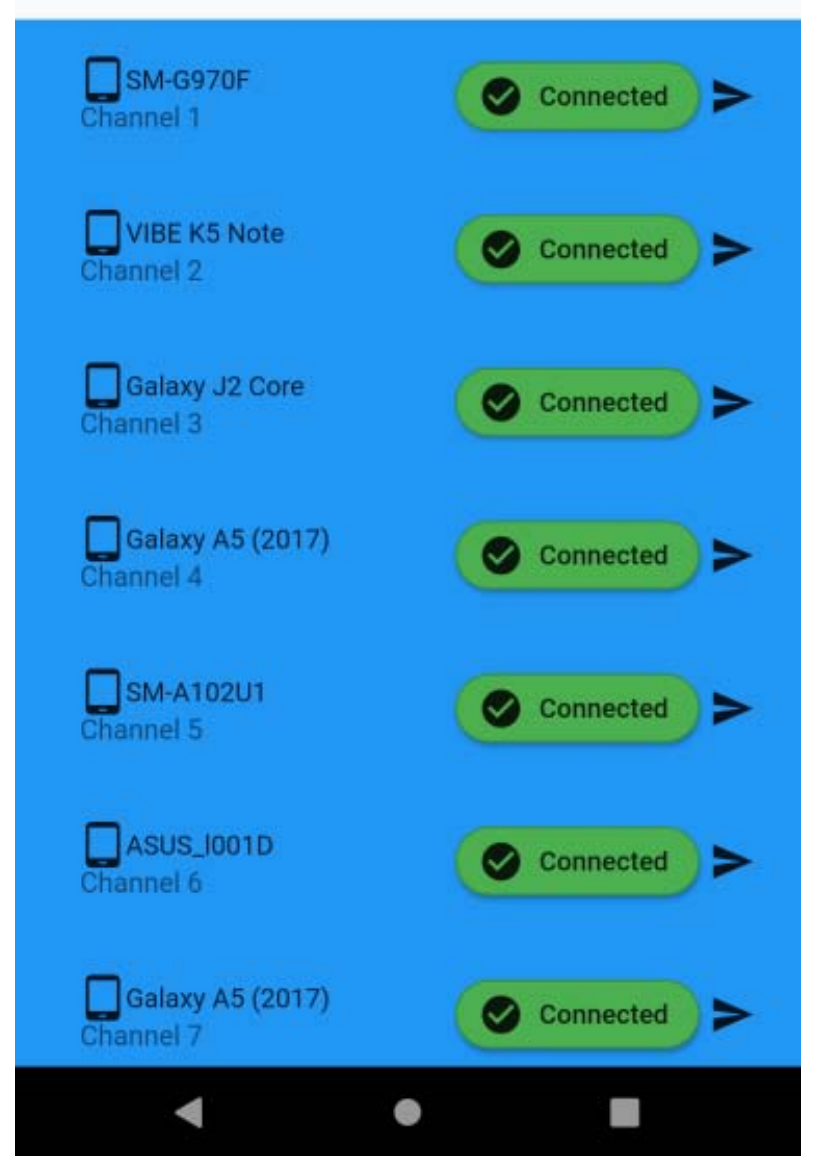

Figure 6: Sharing UUIDs - Approach1 Version2 


\section{Approach 1 - Version 3 (queuing)}

If in its strictest sense the server device must maintain 8 channels irrespective of UUIDs. Then a queue for each channel with an allocation of 1 allotment for a client device would be established, since the UUID would refer to the channel number in which the service is being provided, the queue would be able to guarantee that a connection has already been made with the channel, however it is currently in use and thus, once completed it will become available.

This guarantee stands on the basis that based on the UUID both the client devices would be treated as one device attempting to access the same service. However, it would all come down to which device connects first. Connecting to the server first would be based on which of the 2 devices is nearer, with the device closest, being successful in connecting to the channel and being able to access the channel and the device furthest from the server being successful in connecting to the server but placed into queue. The below figure shows the results of queuing clients with the samw UUID.

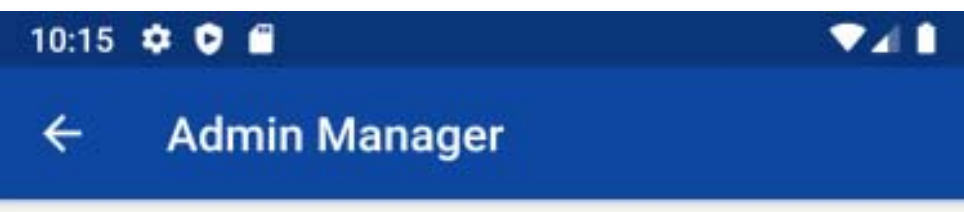

Connected Devices - (4)
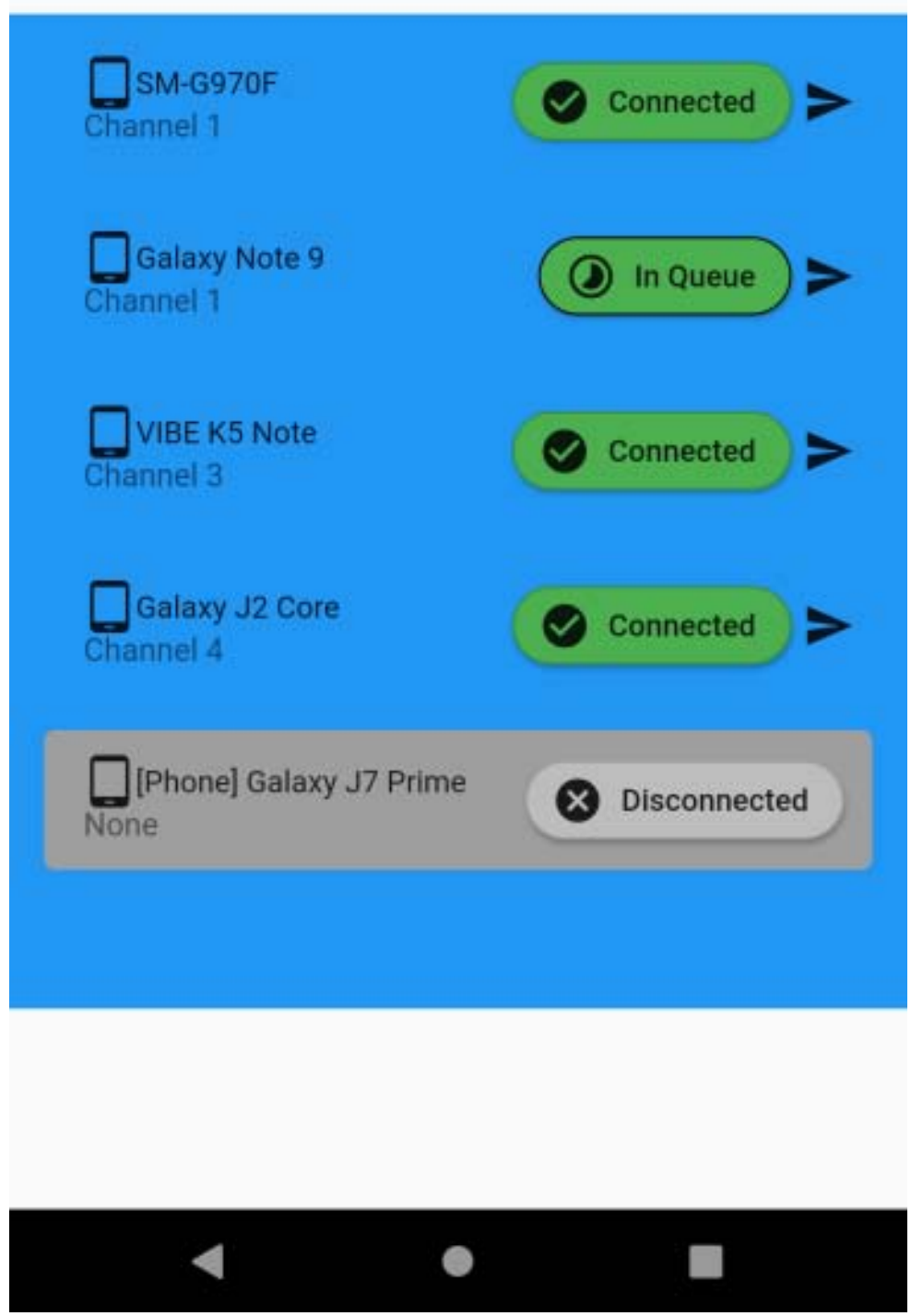

Figure 7: Sharing UUIDs and queuing- Approach1 Version3 
This functionality ensures that the client will receive the quiz content without having to re-attempt connecting to the server multiple times. Thus, this method is a viable option for improving the efficiency of Approach 1.

Approach 2 - Delegate Function()

Approach 2: Given that the server device is mainly responsible for the distribution of quizzing materials and can only supply material to up to 8 devices at a time. The proposed solution would see the modification of the existing system to include the 'delegation' functionality. The delegation functionality works as such: Server Device connects to $8 / 100$ devices at a time, given that the assumed sample/classroom size is 100 students. Then in an effort to increase the efficiency of the system, the Server device can select one of the connected client devices and elevate their privileges or rather give/delegate new functionality to allow the distribution of quiz material. Assuming that the selected client device is able to support the same amount of connections as the server device, this therefore means that a total of $16 / 100$ devices can connect to receive quiz material at a time. If the number of client devices ' $n$ ' that is given Server privileges increases, then the rate in which quiz material can be distributed would take on the form of an exponential curve, thereby increasing the efficiency of the Bluetooth quizzing system.

This approach when compared to the first approach has some immediate advantages: It does not affect the performance of the original hosting device but rather acts as an extension of its functions. What must be taken into consideration is the UUID generator function in which random UUIDs are generated and are hardcoded as the UUID's assigned to the available channels for clients to access their services. This method must ensure that the function delegate() is triggered upon the original Server Device selection of the option to Delegate. As it is an extension of Blue q the interface would consist of the below options.

1. Start Server

2. Manager

3. Data Analytics

4. Delegate

5. Exit

In theory this additional functionality can be likened unto a wi-fi peerto peer network as was postulated by developer.android.com, October2020. It is important to recall that the core concept of a peer-topeer network is to partition tasks or workloads between pairs. In this concept peers are equally privileged, similar to the work done by (SewookJung et al., 2007). However, in this modification of the system, the server device, determines the functions to send to the client device and thus plays an integral role in determining the amount of privileges a selected client device is afforded.
The below Sequence Diagram Shows the flow of events in Approach 1- Version 2: 


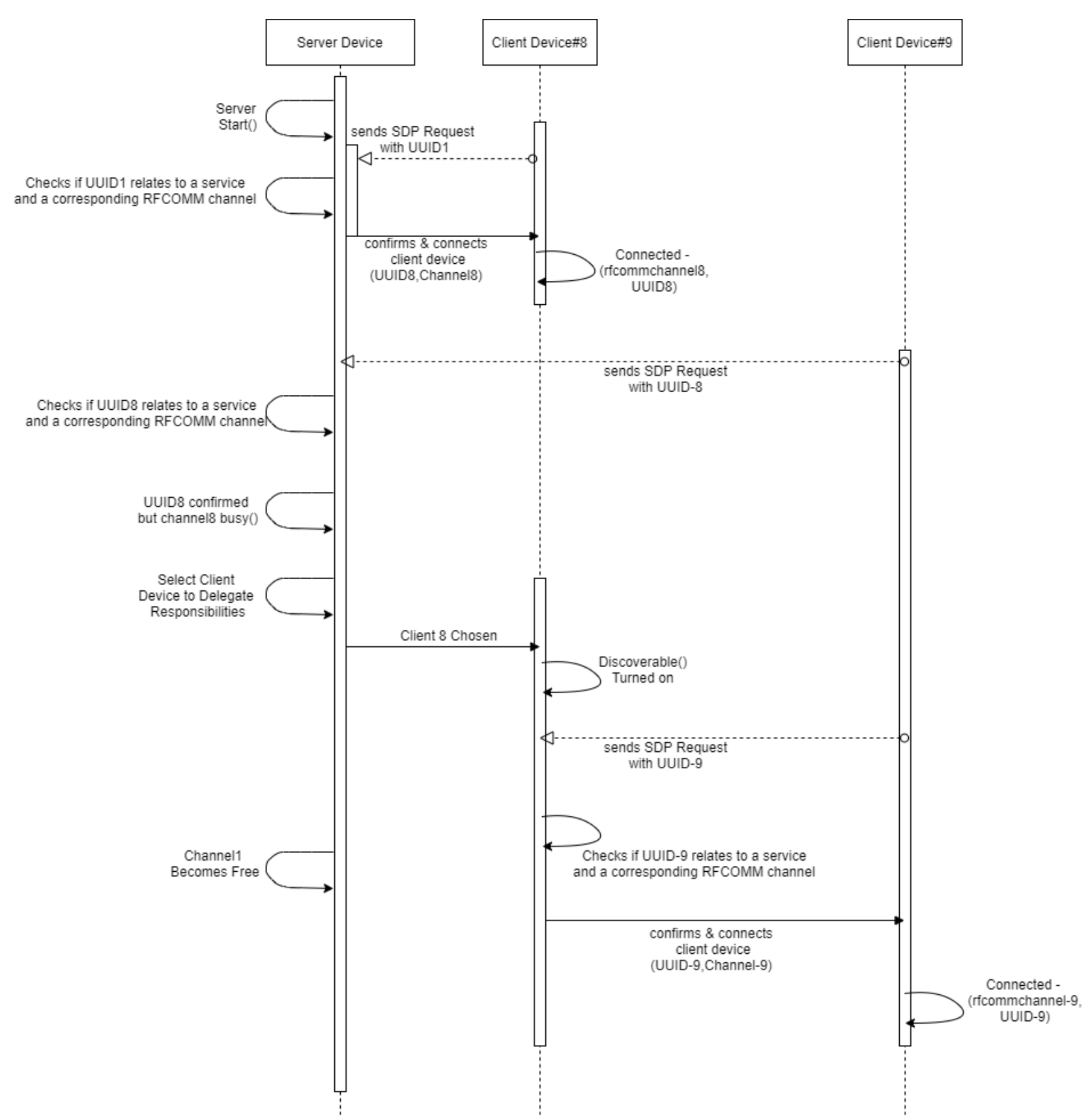

Figure 8: Sequence Diagram - Approach2

\section{Modeling and Testing}

This section presents the results of the testing models implemented and gives an indication as to their individual sample sizes and overall performance against the previous QMS known as Blue Q with respect to simultaneous connections being enabled:

Approach1 version 2 (Shared UUID and multi RFCOMM Channels)

- Total Amount of devices tested $=16$ devices

- Total Amount of devices that can be supported in an instance $=16$ devices

- Average Device Connect Time/s $=2.403 \mathrm{~s}$

- Total Connection Time/s $=38.45 \mathrm{~s}$
Approach1 version 3(Shared UUID and RFCOMM channel queuing)

- Total Amount of devices tested $=16$ devices

- Total Amount of devices that can be supported in an instance $=16$ devices

- Average Device Connect Time/s $=2.403 \mathrm{~s}$

- Total Connection Time $/ \mathrm{s}=38.45 \mathrm{~s}$

Approach 2 (Delegate function)

- Total Amount of devices tested $=16$ devices

- Total Amount of devices that can be supported in an instance $=64$ devices

- Average Device Connect Time/s = 2.329

- Total Connection Time/s $=20$ 
Previous QMS(BlueQ)

- Total Amount of devices tested $=5$ devices

- Total Amount of devices that can be supported in an instance $=5$ devices

- $\quad$ Average Device Connect Time/s $=2.38$

- Total Connection Time/s = 37.3s

From the above results it can be observed that whilst all approaches when compared to the previous
QMS did offer more simultaneous connections, the 'Delegate' approach proved to be the most efficient and scalable method as it was able to support a far greater number of simultaneous connections within approximately the same time as the other proposed solutions. A total of 64 connections versus 16 connections of the other approaches can be supported by the 'Delegate' approach.

\begin{tabular}{|l|r|l|r|r|}
\hline Approaches & $\begin{array}{l}\text { Total Amount devices that can } \\
\text { be supported in an instance }\end{array}$ & $\begin{array}{l}\text { Average Device Connect } \\
\text { Time/s }\end{array}$ & Total Connection Times/s & $\begin{array}{l}\text { Total Amount devices } \\
\text { that can be supported }\end{array}$ \\
\hline $\begin{array}{l}\text { Approach 1 Version 2 - (Shared } \\
\text { UUIDs and Multi RFCOMM }\end{array}$ & 16 & 2.403 & 38.45 & 16 \\
\hline $\begin{array}{l}\text { Approach 1 Version 3 - (Shared } \\
\text { UUIDs and RFCOMM channel }\end{array}$ & 16 & 2.674 & 11.78 & 20 \\
\hline Approach 2 - Delegate & 64 & 2.329 & 37.3 & 16 \\
\hline Previous System - Blue Q & 5 & 2.38 & 54 \\
\hline
\end{tabular}

Figure 9: Testing Model Results for all systems:

A further breakdown on the testing methodology, results and technical analysis can be viewed in the conference paper: "A Technical review of the testing methodologies for QMS Simultaneous Server-Side connections"

\section{Conclusion}

In Conclusion, three solutions/approaches were developed and implemented to solve the problem of lack of popularity or usage of Bluetooth/NFC Quizzing systems within the context of a University Ambient.

These three solutions, saw the usage of the concepts of sharing UUIDs to achieve a greater level of connectivity to the server device as well as the concept of delegating responsibilities from the server device to the client device in an effort to distribute channel sharing load, thus making even more RFCOMM channels available and elevating the privileges of selected clients.

The test results of all approaches were reviewed in an effort to gauge the performance of each of the proposed and developed approaches versus the previous BlueQ system. From the stated results it was shown that the delegate approach proved to be the most meritorious in terms of performance and scalability when compared to the other implemented approaches.

Some of the future work of this paper, would see a similar implementation with the usage of the NFC Protocol. It would also see further work being done in attempting to make the modified QMS a cross-platform entity and not only an 'android specific' solution. Additional work can also be done to further the security aspects surrounding the sharing of UUIDs

\section{References Références Referencias}

1. Ajay, Goyal (May 2019). "NFC vs. Bluetooth: A Detailed Comparison". In: endivesoftware.com.
2. Aneesh, R, B Sreekumari., and K Jiju (Dec 2012). "Design and implementation of Bluetooth MAC core with RFCOMM on FPGA".

3. Brian, Wirsing (2014). "Sending and Receiving Data via Bluetooth with an Android Device". Bär, Henning, Guido Rößling, Erik Tews, and Elmar Lecher. 2006.

4. Davidrajuh, Reggie (January 2009). "Evaluating performance of a Bluetooth based classroom tool". In: International Journal of Mobile Learning and Organization.

5. developer.android.com (October 2020). "Create P2P connections with Wi-Fi Direct".

6. Frydenberg, Jia (2007). "Persistence in University Continuing Education Online Classes". In: he International Review of Research in Open and Distributed Learning 8.3.

7. Grandon, Elizabeth E., Khaled Alshare, and Obyung Kwun (Apr. 2005). "Factors Influencing Student Intention to Adopt Online Classes: A Cross-Cultural Study". In: J. Comput. Sci. Coll. 20.4, 46-56. ISSN: 1937-4771.

8. Hongwei, Du (2013). "NFC Technology: Today and Tomorrow". In: International Journal of Future Computer and Communication 2.4.

9. Ken, Schwaber and Sutherland Jeff (2013). "What is Scrum?"

10. Kulkarni, Chinmay et al. (Dec. 2013). "Peer and Self Assessment in Massive Online Classes". In: ACM Trans. Comput.-Hum. Interact. 20.6. ISSN: 10730516.

11. Lei Wang et al. (2000). "Multipath source routing in wireless ad hoc networks".In: 2000 Canadian Conference on Electrical and Computer Engineering. Conference Proceedings. Navigating to a New Era (Cat. No.00TH8492).Vol. 1, 479-483 vol.1. 
12. McDermott-Wells, P. (2005). "Bluetooth scatternet models". In: IEEE Potentials23.5, pp. 36-39.

13. Michael, Hosein and Bigram Laura (2013). "An Educational Bluetooth Quizzing Application in Android". In: International Journal of Wireless Mobile Networks(IJWMN) 5.6.

14. Miklos, G. et al. (2000). "Performance aspects of Bluetooth scatternet formation". In: 2000 First Annual Workshop on Mobile and Ad Hoc Networking and Computing. MobiHOC (Cat. No.00EX444), pp. 147-148.

15. Mohammed, Salah Abood, Ismail Mahamod, and Rosdiadee Nordin (2016)."A Quiz Management System Based on P2P Near- Field Communication On Android Platform for Smart Class Environments".

16. Naser Hossein, Motlagh (May 2012). "Near Field Communication (NFC) A Technical Overview". In: University of Vasa Faculty of Technology Telecommunication Engeineering - Research Gate.

17. Robert, Triggs (June 2019). "What is NFC and how does it work". In: androidauthority.com.

18. Sewook Jung et al. (2007). "Pervasive and Mobile Computing". In: vol. 3. 6,609-634 vol.3.

19. Shalini, Khemka (December 2018). "What's a Smart Classroom and Why Do You Need It?"

20. Sneh Ratna, Choudhary (March 2020). "Phones compatible with NFC in 2019".

21. Zimbric Frederick J. (Gurnee, IL US) Walsh Michael G. (Johnsburg IL US) Adams Jr. Mark Graham (Hoschton GA US) (Oct 2012). "Methods and Devices for automatic multiple paring of Bluetooth devices". 